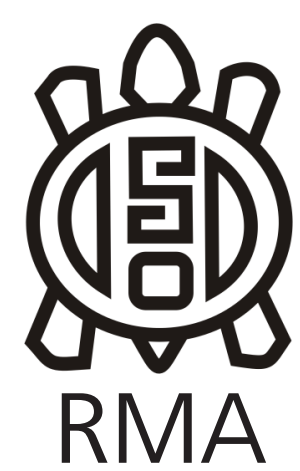

Dossier

\section{Producción lítica en canteras-taller de Antofagasta de la Sierra (Catamarca). Un aporte a la comprensión del registro lítico y su diversidad tecno-tipológica}

\author{
Lithic production in quarry-workshops of Antofagasta de la Sierra \\ (Catamarca). A contribution to understand lithic record and their techno- \\ typological diversity \\ Federico Miguel Bobillo* \\ * Instituto Superior de Estudios Sociales (CONICET). E-mail: fede_bobillo@yahoo.com.ar
}

\title{
Resumen
}

El registro lítico de los contextos de aprovisionamiento es el producto de múltiples actividades y acciones físicas desarrolladas durante milenios de explotación de recursos. Estas prácticas generaron diversas materialidades con atributos tecno-tipológicos distintivos. El objetivo de esta investigación fue conocer los distintos saberes y procedimientos técnicos desarrollados por los artesanos en canteras-taller y su expresión en la cultura material. En este marco, se desarrollaron muestreos y un análisis tecno-tipológico de núcleos, desechos de talla y artefactos formatizados. En primer lugar, se registraron diferentes tipos de núcleos que se relacionan con formas de manipulación de las rocas y "modos de hacer" particulares. En segundo lugar, se reconocieron conjuntos de lascas que se vinculan con la talla de lascas-nucleiformes o con la manufactura de formas-base. Tercero, los instrumentos poseen atributos tipológicos que evidencian acciones físicas en función de crear, y posiblemente utilizar, distintas clases de productos. En conclusión, a partir de la diversidad tecno-tipológica del registro lítico fue posible acceder, por un lado, a una dimensión tecnológica de las actividades de talla en canteras-taller. Por otro lado, se logró documentar distintos "saberes-prácticos" y su expresión en la cultura material.

Palabras clave: Canteras; Aprovisionamiento lítico; Diversidad tecno-tipológica; Estrategias tecnológicas; Artefactos.

\begin{abstract}
Lithic record of the quarry-workshops is the product of multiple activities and physical actions developed during millennia of exploitation of lithic resources. These practices generated materialities with technical-typological attributes that are distinctive. The aim of this research was to know the different knowledge and technical procedures developed by artisans in quarries-workshops and their expression in material culture. In this framework, samplings and a technical-typological analysis of cores, flakes and knapping tools were developed. First, different types of cores were recorded. This are related to particular "ways of doing". In second place, flakes with particular technicaltypological attributes were documented. This are linked to the flakes-cores reductions or to blanks kanpping. Third, knapping tools show physical actions in order to create, and possibly use, different kinds of products. In conclusion, from this research it was possible to access a technological dimension of the activities of knapping activities in quarries-workshop. On the other hand, it was possible to register different "practical knowledge" and its expression in material culture.
\end{abstract}

Keywords: Quarries; Lithic supply; Techno-typological diversity; Technological strategies; Tools.

\section{Introducción}

En el marco de las estrategias tecnológicas vinculadas al aprovisionamiento de recursos líticos en sitios canterataller, se ponen en práctica distintos saberes, planes y procedimientos técnicos (Schiffer y Skibo 1987; Lemonnier 1992; Inizan et al. 1999; Ingold 2008). En la microrregión de Antofagasta de la Sierra (Catamarca) existen canteras-taller de materias primas que fueron intensamente explotadas durante milenios (Aschero et al. 1991; Aschero et al. 2002-2004; Bobillo 2017, 2018; Bobillo y Hocsman, 2015; Escola 2000; Manzi 2006; Pintar 1996, Toselli 1998; entre otros). En el interior de estos sitios, se documentaron contextos líticos cuyas materialidades presentan diversidad en sus atributos tecno-tipológicos. La presente investigación busca realizar un estudio de las prácticas y saberes desplegados por los artesanos en canteras-taller de Punta de la Peña 
(Antofagasta de la Sierra - Catamarca). Para lograr acceder a dicha información se considera relevante profundizar el conocimiento sobre los atributos tecno-tipológicos de los materiales descartados en los palimpsestos que conforman contextos líticos (Bobillo 2017, 2018).

\section{Características de los contextos líticos de las canteras-taller y análisis tecno-tipológico}

En este estudio se consideran los casos de dos canterastaller: Punta de la Peña Zona de Aprovisionamiento y Cantera (en adelante PPZAC) y Pampa Oeste Zona de Aprovisionamiento y Cantera (en adelante POZAC). Las mismas se localizan en una extensa planicie de ignimbrita cubierta por distintos niveles de pedimentación (Tchilinguirian 2008) (Figura 1). En estas canteras, se reconocieron talleres líticos de variada extensión. Por un lado, se documentaron talleres que tienen entre 50 y 80 metros lineales. El registro de los mismos se encuentra compuestos por una gran cantidad de núcleos, desechos de talla y artefactos formatizados que yacen como densos pavimentos líticos. Por otra parte, se reconocieron talleres que poseen entre cuatro y 10 metros lineales de extensión, con menor cantidad de materiales en relación a los talleres extensos. Se trata de eventos de talla discretos y acotadas en el espacio, frecuentes en POZAC, asociados a la explotación de grandes nódulos de vulcanitas que se distribuyen sobre el pedimento de manera irregular.

De acuerdo a los objetivos planteados en la presente investigación, se efectuaron muestreos de distintos talleres líticos en PPZAC y POZAC. Sobre los conjuntos líticos muestreados se realizó un análisis tecno-tipológico de núcleos, desechos de talla y artefactos formatizados. Para efectuar dicho análisis se siguieron los lineamientos propuestos por Aschero $(1975,1983)$ y Aschero y Hocsman (2004). Al respecto, es preciso considerar que en aquellos casos donde no logró definirse una categoría tipológica en la que se enmarcaran determinados productos, se procedió a generar categorías para describir la variabilidad artefactual. Esto fue de particular importancia para definir distintos tipos de núcleos, lascas con atributos tipológicos específicos (como las lascas internas extraídas a partir de la cara ventral de una lasca nodular); e instrumentos que presentan formas de elaboración distintivas (como los perforadores y artefactos burilantes con cuerpo formatizado). En el caso de los núcleos, para definir una categoría tipológica particular se consideró la forma total de la pieza y la articulación de los lascados respecto a las caras y los bordes de las formas-base, como así también la direccionalidad de los negativos de lascado y el tipo de reducción (Aschero 1975; Morello 2005; Barros et al. 2015). Además, se tuvieron en cuenta las cadenas operativas productoras de lascas (sensu Martín Blanco et al. 1994) específicas de cada núcleo.

En relación a los desechos de talla, se identificaron piezas con morfologías particulares que son el producto de acciones técnicas específicas. Este es el caso del conjunto "lascas internas extraídas a partir de la cara ventral de una lasca nodular". Las mismas se definieron considerando: los atributos de la cara ventral (bulbo de percusión pronunciado o difuso, forma de la superficie del talón y presencia/ausencia de estrías y ondas) y los atributos de la cara dorsal de la lasca (cantidad de negativos de lascado y porcentaje de cobertura de corteza) (Aschero 1975, 1983; Espinosa 1995). Por último, para la definición de tipos de instrumentos con atributos específicos, como los perforadores y artefactos burilantes con cuerpo formatizado se tuvo en cuenta las "formas" de cada pieza, previa segmentación en partes y/o sectores diferentes sean filos y/o puntas y/o superficies activas, de acuerdo a atributos estandarizados (Hocsman 2006). La descripción de cada pieza se realizó orientándola de acuerdo al eje de lascado o morfológico; distinguiéndose caras, bordes, dorsos, filos, puntas o superficies activas (Aschero 1975, 1983). El empleo de esta metodología permitió tratar la variabilidad tecnológica y tipológica a nivel del artefacto (Hocsman 2006).

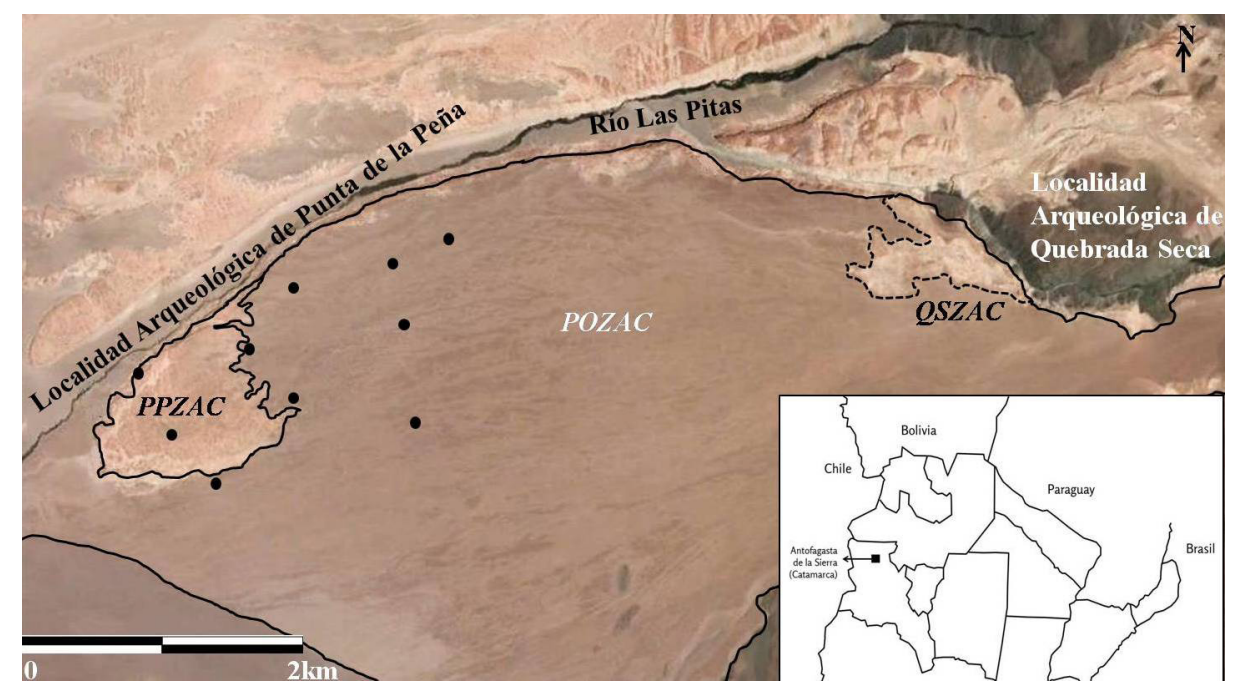

Figura 1. Localización de PPZAC y POZAC y talleres muestreados.

Figure 1. Location of PPZAC and POZAC and sampled workshops 
Tabla 1. Tipos de núcleos registrados en PPZAC y POZAC

Table 1. Cores types registered in PPZAC and POZAC

\begin{tabular}{|c|c|c|}
\hline Tipo de núcleo & $\mathbf{n}=$ & $\%$ \\
\hline Núcleos de lascados aislados & 31 & 13,7 \\
\hline Núcleos bifaciales & 45 & 19,9 \\
\hline Núcleos poliédricos y globulosos & 58 & 25,7 \\
\hline $\begin{array}{c}\text { Núcleos de extracciones unifaciales y bifaciales combinadas } \\
\text { Núcleos de extracciones unifaciales bidireccionales sobre uno o } \\
\text { más frentes de extracción }\end{array}$ & 27 & 11,9 \\
\hline Núcleos piramidal atípico & 12,4 \\
\hline Núcleo discoidal irregular o parcial & 4 & 1,8 \\
\hline Nucleiformes & 1 & 0,4 \\
\hline Fragmento de núcleo no diferenciado & 21 & 9,3 \\
\hline Total & 11 & 4,9 \\
\hline
\end{tabular}

plano (unifaciales) y, generalmente, parten desde dos plataformas de percusión (preparadas o no) situadas en extremos opuestos al frente de extracción, conectándose o superponiéndose en el centro de dicho frente (Figura 2-a) (Bobillo y Aschero 2019). Dicha forma de trabajar los núcleos reflejan acciones y gestiones particulares de los materiales que se tallaron en las canteras-taller. Este tipo de trabajo se registró, también, en la manufactura de perforadores y artefactos burilantes con cuerpo formatizado.

\section{Desechos de talla}

En los contextos líticos de las canteras-taller se hallaron distintas variedades de lascas. Estas se habrían generado en diferentes instancias de la secuencia de reducción de núcleos y formatización de artefactos (Tabla 2). Ahora bien, asociado a estos tipos de lascas se hallaron desechos

\section{El registro lítico de las canteras-taller y su diversidad tecno-tipológica}

\section{Núcleos}

Entre los objetivos de la presente investigación se encuentra estudiar la variabilidad de procedimientos técnicos utilizados para la reducción de núcleos. En este sentido, entre los especímenes muestreados se pudieron reconocer dos grandes categorías desde el punto de vista tecno-tipológico: 1) Núcleos de lascados aislados, núcleos poliédricos, núcleos bifaciales, núcleos piramidales, entre otros; y 2) núcleos que se distinguen y diferencian de los anteriores por presentar un tipo de gestión particular, entre ellos: núcleos con extracciones unifaciales y bifaciales combinadas y núcleos de extracciones unifaciales bidireccionales sobre uno o más frentes de extracción ( $c f$. Bobillo 2018; Bobillo y Aschero 2019) (Tabla 1). A los fines de la presente investigación se hará referencia a este último tipo de núcleos. Los "núcleos de extracciones unifaciales bidireccionales sobre uno o más frentes de extracción" ( $n=28)$ (Tabla 1) presentan formas de manejar las superficies de extracción y las plataformas de percusión que difieren de una talla simple, bifacial, multifacial o centrípeta (Aschero 1975; Boëda 1993; Inizan et al. 1999). En este caso, la articulación de los negativos de lascado respecto a los bordes y las caras de las formas-base (Aschero 1975, 1983) tiende a ser unifacial bilateral, es decir, los negativos de lascado afectan una cara y parten de dos bordes. En efecto, las extracciones bidireccionales -con negativos de lascados paralelos o sub paralelos- se producen sobre un único de las extracciones". Estas lascas se definieron como "lascas internas extraídas a partir de la cara ventral de una lasca nodular" ( $n=32$ ) (Figura 2-b). Las mismas se caracterizan por poseer dos caras ventrales; son lascas que se extraen desde la cara ventral de una lasca nodular, posiblemente grande y espesa (Aschero com. pers. 2018), a la altura del bulbo de percusión. La cara A de la lasca que se extrae, presenta remanentes de una antigua plataforma de percusión (talón) asociados a un bulbo de percusión, estría y ondas. Dichos atributos se corresponden con la cara ventral de la lasca original (nodular) desde la cual se extrajo esta lasca particular. Por otro lado, la cara B, también presenta los atributos de la cara ventral de una lasca: talón, bulbo de percusión, estrías, ondas, entre otros. Entonces, estas lascas se generarían mediante la percusión de una lasca grande o muy grande a la altura del talón, en un proceso de reducción de una lasca nodular (lasca-nucleiforme) (Tabla 1) (Figura 2-c) o formatización de una forma-base. El reconocimiento de "lascas internas extraídas a partir de la cara ventral de una lasca nodular" posibilitó ampliar el conocimiento sobre la variabilidad registrada en los atributos tecno-morfológicos de los desechos de talla en contextos de aprovisionamiento y, de este modo, comprender las acciones e intenciones que les dieron origen.

Al respecto, Escola (2000) registró nucleiformes en una Zona de Aprovisionamiento y Cantera de cuarcita y en sitios residenciales de la microrregión de Antofagasta de la Sierra (Catamarca). Según la propuesta de la autora, con atributos particulares que difieren en cuanto a "origen 
Tabla 2. Tipos de lascas registradas en PPZAC y POZAC

Table 2. Flakes types registered in PPZAC and POZAC

\begin{tabular}{|l|r|r|}
\hline Origen de las extracciones & $\mathrm{n}=$ & \multicolumn{1}{c|}{$\%$} \\
\hline Primaria & 375 & 6.4 \\
\hline Secundaria & 494 & 8.4 \\
\hline Con dorso natural & 67 & 1.1 \\
\hline Flanco de núcleo & 15 & 0.3 \\
\hline Tableta de núcleo & 57 & 1.0 \\
\hline Angular con reserva de corteza & 1909 & 32.5 \\
\hline Angular sin reserva de corteza & 1239 & 21.1 \\
\hline Plana & 78 & 1.3 \\
\hline Angular sin diferenciación de corteza por fractura/s & 658 & 11.2 \\
\hline Adelgazamiento bifacial & 54 & 0.9 \\
\hline En cresta & 2 & 0.03 \\
\hline Reactivación directa & 4 & 0.1 \\
\hline Lasca interna extraída a partir de la cara ventral de una lasca nodular & 32 & 0.5 \\
\hline No diferenciada & 894 & 15.2 \\
\hline Total & 5878 & 100 \\
\hline
\end{tabular}

lascas nodulares (con tamaños mediano-grande y grande) habrían sido transportadas desde las fuentes de aprovisionamiento a los sitios para ser utilizadas como nucleiformes y, así, producir lascas útiles (Escola 2000). En consonancia con estos postulados, los hallazgos de nucleiformes en PPZAC y POZAC, sumado a la presencia de "lascas internas extraídas a partir de la cara ventral de una lasca nodular", evidenciarían la intención de los artesanos de trabajar grandes lascas en los contextos de aprovisionamiento. Estas habrían sido empleadas para extraer otros tipos de lascas, o bien como formas-base de artefactos formatizados (Escola 2000).

\section{Artefactos formatizados}

Como parte de los procesos de elaboración de instrumentos pudieron reconocerse dos trayectorias de producción. Por un lado, se hallaron bifaces en proceso de manufactura $(n=103)$ y puntas de proyectil $(n=9)$ (descartadas por fracturas) que evidencian una tendencia a la elaboración de toolkits relacionados con actividades de caza. Por otra parte, se halló una gran cantidad de instrumentos confeccionados por retalla y retoque marginal, con trabajo no invasivo tanto unifacial como bifacial. Entre los grupos tipológicos registrados se encontraron: cuchillos de filo retocado $(n=38)$, raspadores $(n=97)$, raederas $(n=102)$, cepillos $(n=23)$, cortantes de filo retocado $(n=71)$, gubias $(n=15)$, perforadores $(n=17)$, artefactos burilantes $(n=285)$, entre otros. En relación a los perforadores y artefactos burilantes, se halló un grupo de instrumentos que presentan inversión de trabajo para la adaptación de su parte prensil (sector pasivo). A raíz de este atributo distintivos, estas piezas se denominaron como "perforadores y artefactos burilantes con cuerpo formatizado" $(n=11)$.

En este tipo de piezas, la conformación del sector pasivo posibilita la presión del artefacto y el uso de una punta activa (Figura 2-d). Un rasgo tipológico distintivo es la forma en la que se trabajó el sector prensil, ya que el patrón de retalla y retoque posee similitud con el patrón de extracción de lascas registrado en núcleos de extracciones unifaciales bidireccionales sobre uno o más frentes de extracción: negativos de lascado que se extienden desde dos plataformas de percusión opuestas y que presentan un patrón de extracciones bidireccionales, paralelas 0 sub paralelas, sobre un único plano (Figura 2-a). La similitud en el tratamiento dado a los perforadores y artefactos burilantes con cuerpo formatizado y núcleos de extracciones unifaciales bidireccionales sobre uno o más frentes de extracción permite pensar en un "modo de hacer" particular relacionado no sólo con los procesos de reducción de núcleos y extracción de formas-base, sino también con la formatización de instrumentos para ser utilizados en actividades de punción y/o incisión (sensu Aschero 1975, 1983). En futuras investigaciones se abordará en profundidad estos modos de hacer particulares, buscando identificar trayectorias de producción entre ambas clases tipológicas que implicaran procesos de reciclaje o requerimientos en la formatización de formas-base (Hocsman y Escola 2006-2007).

\section{Discusión y conclusiones}

Los datos obtenidos en este trabajo constituyen un aporte al conocimiento de la diversidad tecno-tipológica de los materiales líticos que integran los contextos de canteras-taller en Antofagasta de la Sierra (Catamarca) a nivel microregional y de la Puna Argentina a escala regional. El registro lítico de estos sitios informa sobre procedimientos particulares vinculados con la reducción de núcleos y formatización de formas-base. En primer lugar, logró reconocerse distintos procedimientos y modos de manipular las rocas para extraer formas-base. Entre los métodos de reducción de núcleos se identificó técnicas de talla simple, multifacial, bifacial y centrípeta (Aschero 1975; Boëda, 1993; Inizan et al. 1999), además 
Figura 2. a. Núcleos de extracciones bidireccionales sobre un mismo frente de extracción. b. Lascas internas extraídas a partir de la cara ventral de una lasca nodular. c. Nucleiforme sobre lasca nodular: vista de la cara ventral (A) y vista de la cara dorsal (B). d. Artefacto burilante con cuerpo formatizado.

Figure 2. a. Bi-directional extractions on the same extraction front cores. b. Internal flakes extracted from the ventral face of a nodular flake. c. flake-core on a nodular flake: view of ventral face (A) and view of dorsal face (B). d. buring devices with shapped body.

de piezas que presentan gestiones particulares como ser los núcleos de lascados unifaciales y bifaciales combinados y los núcleos de extracciones unifaciales sobre uno o más frentes de extracción. Esta diversidad de prácticas y saberes da cuenta de formas de conceptualizar

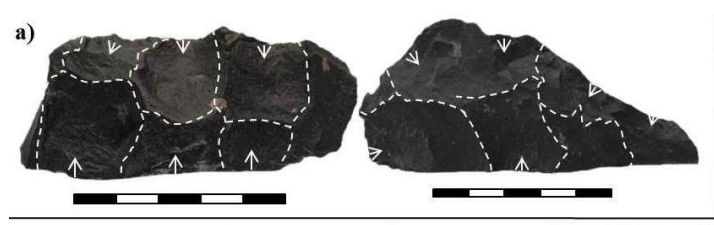
b)

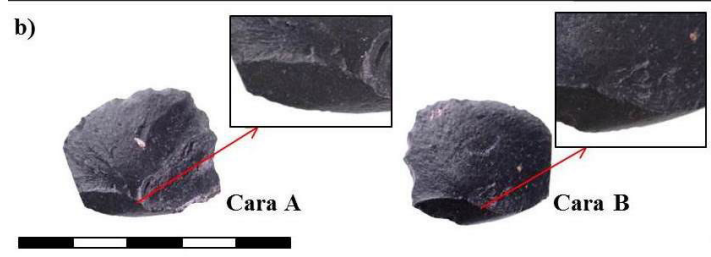

d)
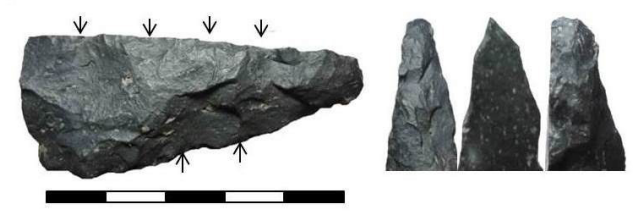

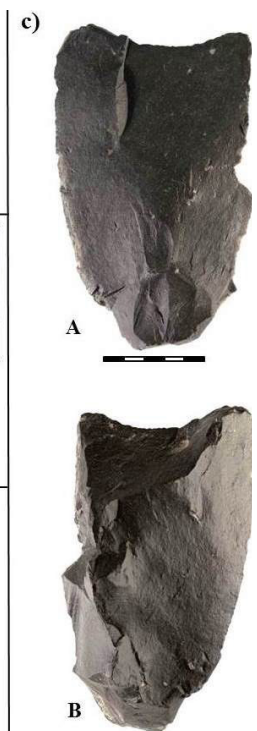
y manejar los soportes de distintas maneras, además de habilidades particulares puestas en funcionamiento para extraer formas-base (Lemonnier 1992; Ingold 2008).

En segundo lugar, se documentaron desechos de talla diagnósticos de acciones específicas. El hallazgo de lascas internas extraídas a partir de la cara ventral de una lasca nodular se vincularía con procesos de reducción de lascas-nucleiformes y/o formatización de formas-base. Al respecto, Colombo (2013) registró este tipo de actividades en canteras de cuarcita del centro de Tandilia. En estos contextos líticos, las lascas de descortezamiento se utilizaron como lascas nodulares para extraer otro tipo de lascas (Colombo 2013). Estas últimas tendrían atributos similares a las lascas internas extraídas a partir de la cara ventral de una lasca nodular.

Tercero y último, se registraron distintos grupos tipológicos. Específicamente, los perforadores y artefactos burilantes con cuerpo formatizado presentan un patrón de trabajo similar al documentado en los núcleos de extracciones unifaciales bidireccionales sobre uno o más frentes de extracción. Estas semejanzas entre ambas clases tipológicas estarían indicando modos de trabajar las rocas que son similares, pero con finalidades distintas. En este sentido, se trataría de un corpus de saberes-prácticos semejantes (Schiffer y Skibo 1987; Ingold 2008) donde se mantiene un mismo concepto de trabajo para dar origen a diferentes productos.

En conclusión, en las canteras-taller de Punta de la Peña (Antofagasta de la Sierra-Catamarca) se logró registrar distintos saberes, prácticas y modos de hacer y su expresión en la cultura material. El estudio tecnotipológico de instrumentos y otras clases tipológicas, asociado a un análisis de las materias primas y acciones físicas (Inizan et al. 1999), posibilitó comprender el conjunto de conocimientos utilizados por los artesanos

para crear productos en canteras-taller (Schiffer y Skibo 1987).

\section{Agradecimientos}

A Carlos Aschero y Salomón Hocsman por los aportes realizados durante el análisis de los conjuntos líticos. A Marcela Alonso por la fotografía de los materiales. A los evaluadores por sus comentarios y sugerencias. Este trabajo se realizó en el marco de los proyectos PIUNT G503 y PIP-CONICET 577 (dirigidos por Carlos A. Aschero) y PICT 1703 (dirigido por Salomón Hocsman).

\section{Referencias citadas:}

Aschero, C. A. (1975). Ensayo para una clasificación morfológica de artefactos líticos aplicada a estudios tipológicos comparativos. Informe al CONICET. Manuscrito inédito.

Aschero, C. A. (1983). Ensayo para una clasificación morfológica de artefactos líticos aplicada a estudios tipológicos comparativos. Apéndices A-C. Manuscrito inédito.

Aschero, C. A., Elkin, D. y Pintar, E. (1991). Aprovechamiento de recursos faunísticos y producción lítica en el Precerámico Tardío. Un caso de estudio: Quebrada Seca 3 (Puna Meridional Argentina). Trabajo presentado en el XI Congreso Nacional de Arqueología Chilena. Santiago de Chile, Chile.

Aschero, C. A., Escola, P. S., Hocsman, S. y Martínez, J. (2002-2004). Recursos líticos en la escala microregional Antofagasta de la Sierra. Arqueología, 12, 9-36.

Aschero, C. A. y Hocsman, S. (2004). Revisando cuestiones 
tipológicas en torno a la clasificación de artefactos bifaciales. En A. Acosta, D. Loponte y M. Ramos (Eds.). Temas de Arqueología. Análisis Lítico (pp. 7-25). Universidad Nacional de Luján: Luján.

Barros, M. P., Messineo, P. G. y Colantonio, M. J. (2015). Chert quarries and workshops in the Humid Pampa subregión: New contributions on exploitation techniques and circulation through study of chaînes opératoires. Quaternary International, 375, 99-112.

Bobillo, F. M. (2017). Estudio comparativo de Zonas de Aprovisionamiento y Cantera (ZAC) de Punta de la Peña (Antofagasta de la Sierra, Catamarca): análisis de las actividades de talla en una cantera y cantera-taller. Intersecciones en Antropología, 18, 67-77.

Bobillo, F. M. (2018). Estrategias tecnológicas empleadas en la explotación de materias primas y formatización de artefactos en Pampa Oeste Zona de Aprovisionamiento y Cantera (Antofagasta de la Sierra - Catamarca, Argentina). Chungara, Revista de Antropología Chilena, 50 (2), 255-267.

Bobillo, F. M. y Hocsman, S. (2015). Mucho más que solo aprovisionamiento lítico: actividades en canteras y prácticas sociales en las fuentes de Pampa Oeste, Quebrada Seca y Punta de la Peña (Antofagasta de la Sierra, Catamarca). Revista del Museo de Antropología, 8, 23-44.

Bobillo, F. M. y Aschero, C. A. (2019). Prácticas de reducción de núcleos en Punta de la Peña (Antofagasta de la Sierra, Catamarca): un análisis de los distintos modos de trabajar las rocas en contextos de aprovisionamiento. Arqueología, 25 (1), 103-127.

Boëda, E. (1993). Le débitage discoïde et le débitage Levallois récurrent centripède. Bulletin de la Société préhistorique française, 90(6), 392-404.

Colombo, M. (2013). Los cazadores recolectores pampeanos y sus rocas. La obtención de materias primas líticas vista desde las canteras arqueológicas del centro de Tandilia (Tesis doctoral inédita). Facultad de Ciencias Naturales y Museo, Universidad Nacional de La Plata, La Plata.

Escola, P. S. (2000). Tecnología lítica y sociedades agropastoriles tempranas (Tesis doctoral inédita). Facultad de Filosofía y Letras. Universidad Nacional de Buenos Aires, Buenos Aires.

Espinosa, S. (1995). Dr. School y MonsierFleur: de Talones y Bulbos. Cuadernos Instituto Nacional de Antropología y Pensamiento Latinoamericano, 16, 315- 328.

Hocsman, S. (2006). Producción lítica, variabilidad y cambio en Antofagasta de la Sierra (5500-1500 AP). (Tesis Doctotal inédita), Universidad Nacional de La Plata, Argentina.

Hocsman, S. y Escola, P. S. (2006-2007). Inversión de trabajo y diseño en contextos líticos agro-pastoriles (Antofagasta de la Sierra, Catamarca). Cuadernos del Instituto Nacional de Antropología y Pensamiento Latinoamericano, 21, 75-90.

Ingold, T. (2008). Tres en uno: cómo disolver las distinciones entre cuerpo, mente y cultura. En T. SánchezCriado (Ed.). Tecnogénesis. La construcción técnica de las ecologías humanas (pp. 1-34). Madrid: Antropólogos Iberoamericanos en Red.

Inizan, M. L., Reduron-Ballinger, M., Roche, H. y Tixier, J. (1999). Technology and Terminology of Knapped Stone. Préhistoirede la Pierre Taillée. Nanterre: CREP.

Lemonnier, P. (1992). Elements for an Anthropology of Technology. Ann Arbor: Museum of Anthropology, University of Michigan.

Manzi, L. (2006). Estrategias y formas de uso del espacio en poblaciones cazadoras recolectoras de la Puna meridional argentina. Oxford: BAR International Series 1465.

Martín Blanco, P., Jimenez Manzanares, A., Sanguino González, J. y Gómez Laguna, A. J. (1994). Identificación de cadenas operativas líticas en el sitio arqueológico de "Xasa de la Mina II" (Argamasilla de Alba, c. Real). Consideraciones acerca de los yacimientos superficiales sin contexto estratigráfico. Zephyrvs, XLVII, 15-40.

Morello, F. (2005). Tecnología y métodos para el desbaste de lascas en el norte de Tierra del Fuego: los núcleos del sitio Cabo San Vicente. Magallania, 33(2), 29-56.

Pintar, E. (1996). Prehistoric Holocene Adaptations to the Salt Puna of Northwestern Argentina. (Tesis doctoral inédita), Southern Methodist University, Estados Unidos.

Schiffer, M. B. y Skibo, J. M. (1987). Theory and Experiment in the Study of Technological Change. Current Anthropology, 28, 595-622.

Tchilinguirian, P. (2008). Paleoambientes holocénicos en la puna austral, provincial de Catamarca $\left(27^{\circ} \mathrm{S}\right)$ : Implicancias geoarqueológicas (Tesis doctoral inédita), Universidad Nacional de Buenos Aires, Argentina.

Toselli, A. (1998). Selección de materias primas líticas y organización tecnológica en el sitio Punta de la Peña 4 (PP4), Depto. Antofagasta de la Sierra, Prov. de Catamarca. (Tesis de licenciatura inédita), Universidad Nacional de Tucumán, Tucumán. 